\title{
Le patrimoine militaire sur la scène urbaine : les processus de reconversion des sites militaires à Metz
}

Military heritage in the city: restructuring processes of former military lands in Metz

Die städtische Inszenierung des militärischen Erbes : Umstellungsprozesses der militärischen Liegenschaften in Metz

Marie-Caroline Gaymard

\section{OpenEdition}

\section{Journals}

Electronic version

URL: http://journals.openedition.org/rge/5330

DOI: $10.4000 /$ rge.5330

ISSN: 2108-6478

\section{Publisher}

Association des géographes de l'Est

Printed version

Date of publication: 30 December 2014

ISSN: 0035-3213

\section{Electronic reference}

Marie-Caroline Gaymard, « Le patrimoine militaire sur la scène urbaine : les processus de reconversion des sites militaires à Metz », Revue Géographique de l'Est [Online], vol. 54 / n³-4 | 2014, Online since 11 February 2015, connection on 08 September 2020. URL : http://journals.openedition.org/rge/5330 DOI : https://doi.org/10.4000/rge.5330

This text was automatically generated on 8 September 2020

Tous droits réservés 


\section{Le patrimoine militaire sur la scène urbaine : les processus de reconversion des sites militaires à Metz}

Military heritage in the city: restructuring processes of former military lands in Metz

Die städtische Inszenierung des militärischen Erbes : Umstellungsprozesses der militärischen Liegenschaften in Metz

Marie-Caroline Gaymard

1 Lorsque Nicolas Sarkozy, dans son discours aux armées du 8 janvier 2010, affirme : «L'armée française est en charge de la sécurité des Français, pas de l'aménagement du territoire», l'ancien président de la République a une vision partielle du rapport entre l'institution militaire et l'espace français métropolitain. Bien que ce ne soit pas sa vocation première, il est clair que l'institution militaire joue un rôle majeur dans certaines régions, tant par ses retombées économiques que par son emprise spatiale au cœur de qu'on appelle les «villes de garnison» comme le sont ou l'étaient Besançon, Angers, Nîmes, Pau, Clermont-Ferrand, Poitiers...

2 Les réformes militaires ont un impact fort sur le patrimoine immobilier des armées, impliquant des modifications d'usage et la libération d'emprises qui, une fois vides, deviennent cessibles. Ce faisant, la mutation des armées accélère la transformation des villes françaises (Leroux, 2003). Pour accompagner ces changements, l'institution militaire a donc développé des compétences juridiques définissant une politique d'ensemble en matière immobilière et foncière, via lesquelles elle renforce sa position d'acteur du développement des territoires.

3 Dans ce contexte, notre travail se concentre sur l'agglomération de Metz. La présence militaire a marqué profondément l'espace urbain, ce qui pousse aujourd'hui les acteurs institutionnels à développer des stratégies destinées à changer son image de ville de 
garnison. La création de la Communauté d'Agglomération Metz-Métropole (CA2M) en 2002, regroupant 40 communes voisines de Metz, s'est faite autour de projets d'envergure destinés à faire de la ville une véritable métropole régionale : renouvellement urbain autour du nouveau quartier de l'Amphithéâtre à proximité du Centre Pompidou-Metz et du futur Centre des Congrès, réalisation du CHR MetzThionville sur l'ancien terrain militaire de Mercy, aménagement du site militaire du Mont-Saint-Quentin en poumon vert de l'agglomération, projet de transport en commun Mettis... Parallèlement, dans le cadre de Metz 2030, projet de développement à l'échelle de la ville soutenu par la municipalité, l'avenir se fonde sur la culture, la relance démographique et l'enseignement supérieur. L'économie de la ville étant à $80 \%$ présentielle - elle regroupe les activités locales de production de biens et de services à destination des personnes présentes dans l'agglomération messine, qu'elles soient résidentes ou touristes -, le départ de la population équivaut à un manque à gagner économique de taille. Ce programme vise donc à faire de Metz une ville verte à haute qualité de vie, au bassin d'emploi attractif et renouvelé, concentrant tous les attributs d'une métropole : capitale politique, centre économique et foyer culturel (Bonnet, 1994). La ville profite ici d'une lisibilité nationale et européenne accrue depuis l'ouverture réussie du Centre Pompidou-Metz en 2010, qui a déjà accueilli plus d'un million de visiteurs (Marquis \& Demange, 2010).

L'exemple de Metz est particulièrement pertinent car l'histoire de la ville, qui a pris naissance sur un site naturel défensif à proximité des frontières, est mêlée à celle des armées. Quatre systèmes fortifiés se dégagent : le mur linéaire romain, le tracé bastionné de l'époque Cormontaigne, les forts détachés Séré de Rivières et les Festen de la 2e moitié du XIXe siècle et enfin la ligne Maginot (Turrel, 1986). Elle présente donc un échantillonnage représentatif des formes que prend le patrimoine militaire et des reconversions qui ont été menées en France au cours des 40 dernières années. En Moselle, les villes militaires peuvent être classées comme «ville citadelle» du XVIIe siècle, «ville des quartiers militaires» (ou ville de garnison) de la fin du XIXe siècle ou «ville des formes militaires éclatées», autant de générations successives de modèles urbains modelés par la présence militaire (Mathis, 2011). Metz, par son histoire et sa position de défense des frontières orientales du territoire, est bien une ville citadelle ; mais l'évolution de la trame urbaine la classe plus spécifiquement comme ville de garnison.

5 On comprend alors l'enjeu que représente l'annonce des restructurations de 2008. Le redéploiement des infrastructures militaires aurait occasionné pour l'agglomération une perte de 5650 emplois militaires et civils, soit 12\% des suppressions envisagées sur le territoire national (INSEE, 2011). Sur les sept communes directement impactées par la réforme de la carte militaire (Metz, Augny, Châtel-Saint-Germain, Marly, Montignylès-Metz, Moulins-lès-Metz et Woippy), le redéploiement des régiments est à l'origine d'un profond malaise identitaire, économique et démographique, qui s'ajoute au déclin de son activité industrielle. Alors que jusque-là, les libérations des terrains se faisaient progressivement, la cession de plusieurs centaines d'hectares au même moment en fait un cas atypique. Ce caractère multisite gêne la mise en place d'une vision partagée entre les communes concernées.

Cet article interroge donc les conditions selon lesquelles la reconversion des emprises devient intéressante, alors qu'elle ne peut être rapidement intégrée aux processus de recomposition territoriale auxquels participent aménageurs et urbanistes. La question 
d'une géographie urbaine des emprises militaires se pose : quelles sont les conséquences de l'évolution de la présence militaire dans les projets d'aménagement de l'agglomération messine et comment les nouveaux usages du patrimoine militaire urbain se donnent-ils à voir au sein de la métropolisation du territoire ? Pour répondre, il faut appréhender la mise en place d'une stratégie immobilière et foncière spécifique, en considérant les mécanismes inhérents aux réformes du service public et la spécificité du patrimoine militaire.

7 Sont ici retranscrits les résultats d'une démarche d'enquêtes et d'observations lors d'un stage à la Sous-Direction de l'Immobilier et de l'Environnement au Ministère de la Défense à Paris, entre février et avril 2013. 16 entretiens ont eu lieu au cours de cette période, auprès des responsables techniques et politiques en charge de l'aménagement et de l'urbanisme (Ville de Metz, CA2M) et des personnels militaires en charge des affaires foncières.

\section{L'évolution de la présence militaire dans la ville}

\section{A. Un contexte de réformes}

C'est dans l'histoire que se trouvent les origines des mutations territoriales qu'a connu l'armée française au cours des vingt dernières années. La fin de la Guerre Froide rend obsolète la concentration de moyens dans le nord-est de la France. Le Plan Armées 2000 du 22 août 1990 annonce donc une diminution d'un quart des effectifs, la réduction de la durée du service militaire et le repli des forces stationnées en Allemagne. Des villes telles que Metz, Nancy, Épinal, où la fonction militaire était omniprésente, entrent dans une phase d'adaptation : la notion même de «ville de garnison» s'étiole (Merchet, 2005).

L'année 2008 marque un nouveau tournant. La politique de défense ne dépend plus seulement de données stratégiques et de traits propres à l'organisation militaire de la France, mais s'inscrit dans la Révision Générale des Politiques Publiques (RGPP) de 2007. Cette dernière concerne l'ensemble des administrations publiques et vise la maitrise des dépenses des services publics, d'où la mise en place de réformes qui touchent autant la carte judiciaire que les réseaux financiers locaux de l'État.

Concernant le fait militaire, la réforme s'appuie sur le Livre Blanc de 2008 qui définit une nouvelle stratégie de défense et de sécurité nationale. Il conduit à un resserrement continu de l'implantation territoriale des armées : d'ici la fin 2015 disparaissent 82 unités dont 10 bases aériennes et une base aéronavale, sans compter les services déconcentrés d'administration'2. Pour l'appliquer, trois principes structurent l'action immobilière et foncière du Ministère de Défense : la mutualisation, qui se traduit par le groupement de toute l'administration centrale du ministère à Balard, dans le XVe arrondissement de Paris ; la densification, qui se traduit par le regroupement des forces stationnées sur le territoire métropolitain sous formes de «Base de Défense»; et la rationalisation, qui se traduit par une simplification des processus d'achat et de vente des emprises ${ }^{3}$. La création d'une Base de Défense (BdD) sur le site messin, à la place de l'ancienne Zone de Défense de la Région Terre Nord-Est, assure à la ville la continuité de la présence militaire : son importance stratégique perdure dans le temps. La BdD crée une chaine administrative unique à l'échelle locale, assurant une liaison horizontale entre les différentes formations implantées dans le secteur de responsabilité de la BdD. De fait, les mesures de réorganisation de l'outil militaire 
placent le ministère de la Défense au cœur d'un débat multidimensionnel sur la gestion du territoire.

\section{B. Des emprises considérables et hétérogènes}

11 La présence militaire a pesé sur l'urbanisme messin. La ville étant embastionnée au cœur d'une enceinte fortifiée qui s'ouvre sur des portes monumentales (Porte des Allemands), le périmètre urbain est réduit et très dense. Le patrimoine étudié appartient à des époques différentes : au fil des siècles, les bâtiments militaires ont formé un ensemble d'une grande diversité, à vocation opérationnelle ou administrative, qui divergent par leur localisation, leur surface et leur aménagement. Dans le centre-ville, les constructions en pierre de Jaumont constituent un patrimoine militaire de qualité, témoin de l'idéologie royale et de la puissance militaire de la ville (Bour, 2007), souvent classé monument historique. Dans les espaces périurbains, terrains d'exercice, fortifications, casernes, locaux d'habitation, bases aériennes, dépôts de toutes dimensions peuvent être déclassés puis aliénés. Vendre et reconvertir ce patrimoine entraîne donc une variété de processus complexes, car la valeur des emprises est inégale. Les deux cartes suivantes, qui présentent l'historique des libérations et reconversions menées sur le territoire de la CA2M pour la première et les projets de la CA2M pour la seconde, illustrent bien la difficulté de mener des programmes d'acquisition foncière et d'aménagement à la fois cohérents et consensuels au vu de l'ampleur des libérations en cours et de la diversité des emprises (en rouge sur la carte), dans le cadre d'une métropolisation déjà bien avancée.

Figure 1 : Carte de l'historique des reconversions militaires sur la CA2M

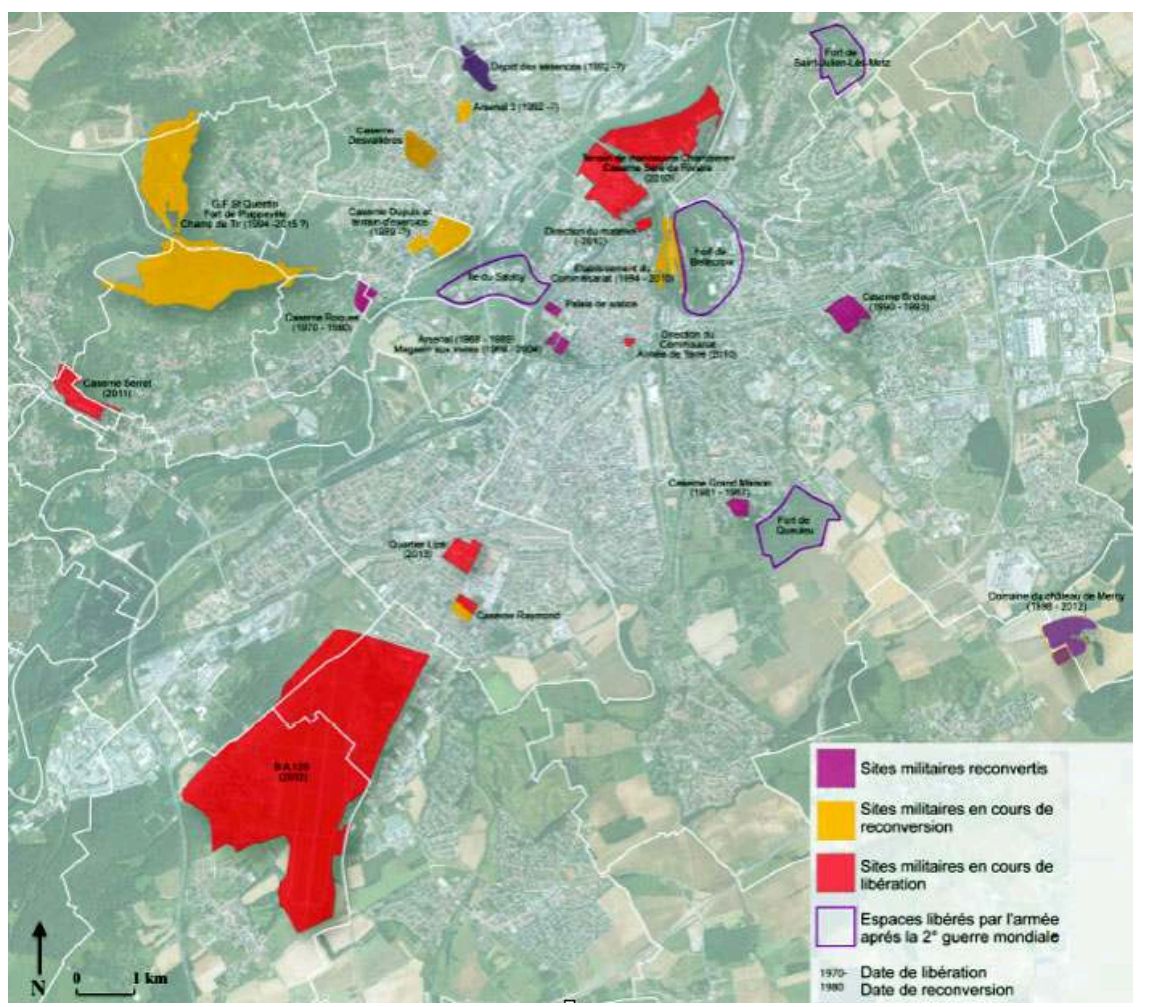

Source : DAR, 2010 ; actualisation : Marie-Caroline Gaymard, 2013 


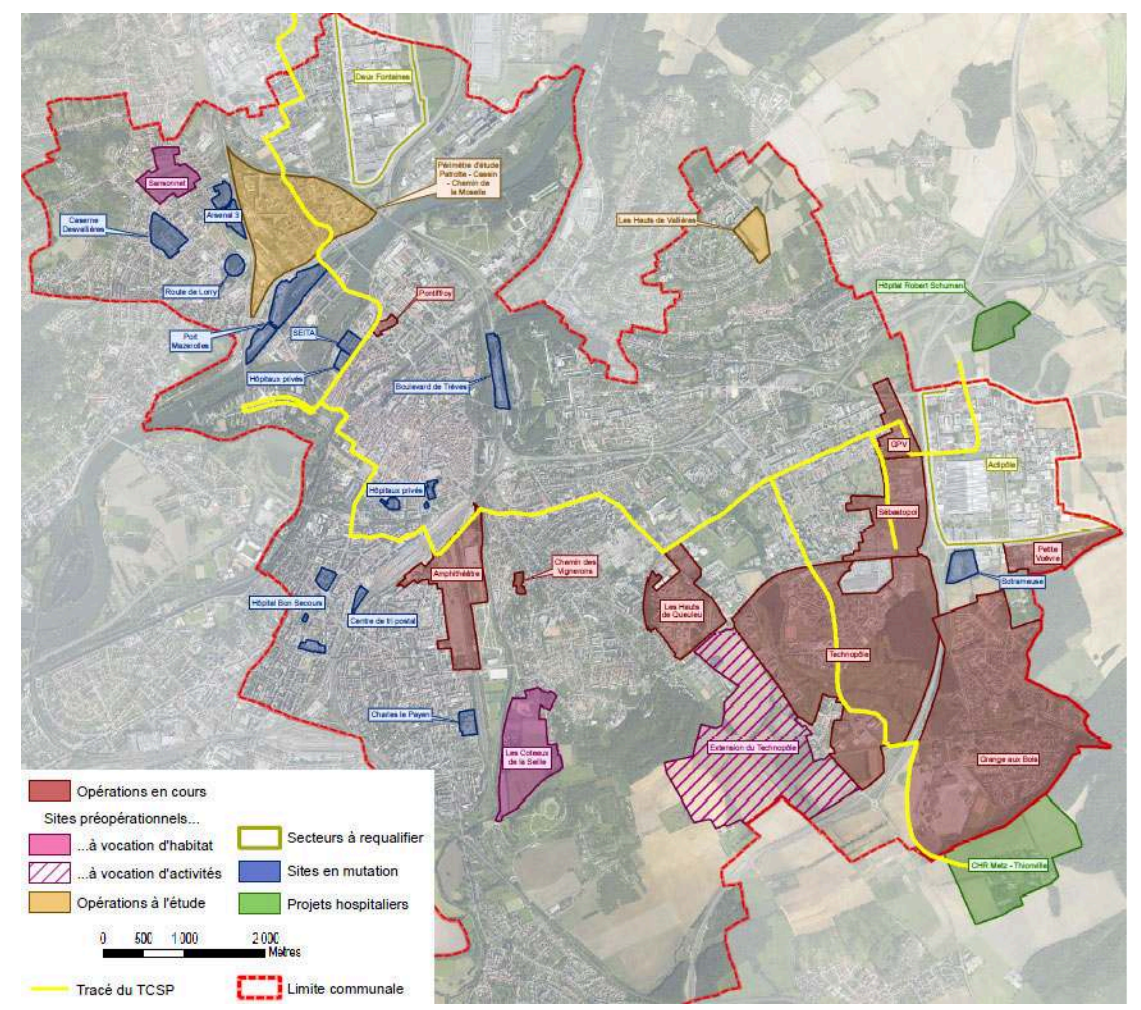

Source : Ville de Metz, 2013

Le patrimoine de l'armée de terre $-70 \%$ de la superficie totale des emprises militaires - se démarque par sa localisation essentiellement urbaine. En effet, l'étalement urbain depuis la fin de la Seconde Guerre Mondiale a rapproché les emprises militaires des villes. Les quartiers militaires (logements, hôpitaux, terrains d'exercice) construits en périphérie sont ainsi encerclés par le bâti plus récent, formant des entités de taille considérable dans les franges urbaines (Guelton, 2001). Ainsi, à Metz, 7\% de la superficie totale de l'agglomération est toujours occupée par des quartiers militaires qui forment des éléments de ruptures dans le tissu urbain (Gallas et al, 2009). Par le biais de vidéosurveillance, grilles, gardes à l'entrée et murs, ils présentent peu d'accès à la vie civile. L'emprise militaire est une terra incognita, un symbole mystérieux qu'on côtoie à défaut d'y entrer.

La fermeture des emprises militaires à la ville est renforcée par leur statut particulier. Elles constituent des zones inconstructibles qui relèvent d'autorités distinctes de la commune et de législations différentes qui génèrent des contraintes fortes sur l'usage des sols : les municipalités n'ont aucune prise politique sur elles. Alors même que le Plan Local d'Urbanisme (PLU) tient compte des règles de la défense nationale, elles ne peuvent prévoir le destin urbain de ces terrains et n'ont qu'un rôle de gestion de l'urgence, dans le sens où leur action se limite généralement à une logique de réaction par rapport aux décisions du Ministère de la Défense. Les différentes échelles d'intervention créent une confusion que tente d'atténuer l'interaction des acteurs à tous les niveaux de compétence (préfet, maire, négociateur de la MRAI, commandant de BdD). 
14 Véritable ville dans la ville, l'emprise militaire doit donc être réintégrée à l'espace public civil et retrouver une fonctionnalité en adéquation avec le tissu urbain.

\section{Des contraintes qui restreignent les changements d'usage}

Les obstacles à la reconversion des sites militaires sont nombreux, de la superficie démesurée des sites à la pollution pyrotechnique, aux contraintes d'accès, de réseaux, de servitudes et celles liées au type de bâti (hauteur des plafonds, ouverture des fenêtres, non-conformité aux normes en vigueur, ruines). Ainsi, tous les projets élaborés sur les sites militaires visés par la politique de reconversion n'ont pas pu se concrétiser (Pommier, 2010).

Les raisons sont multiples. Par exemple, en milieu urbain dense et prospère, la valeur potentielle de l'emprise est grande. Celles dont le coefficient d'occupation des sols est supérieur à 1 (immeubles) sont donc vendues aux mairies ou aux établissements publics à des prix négociés car, malgré leur situation en centre-ville, leur structure technique en fait des bâtiments très complexes à réaménager (Guelton, 1998) : la reconversion du Magasin aux Vivres a pris près de 40 ans. Dans les communes périurbaines, la reconversion est l'occasion de rechercher de nouvelles opportunités avec les responsables économiques (Godet, 2007), à condition que la demande pour de nouveaux équipements ou services soit suffisante pour que l'opération soit rentable. Enfin, les terrains en état de nature, dont la valeur architecturale et foncière est très faible et le potentiel peu intéressant (fortifications de Bellecroix illustrés en photo 1) rentrent dans les réserves foncières municipales dans l'attente d'un projet viable.

Photo 1 : Fortifications de Bellecroix, au nord des casernes du Boulevard de Trèves

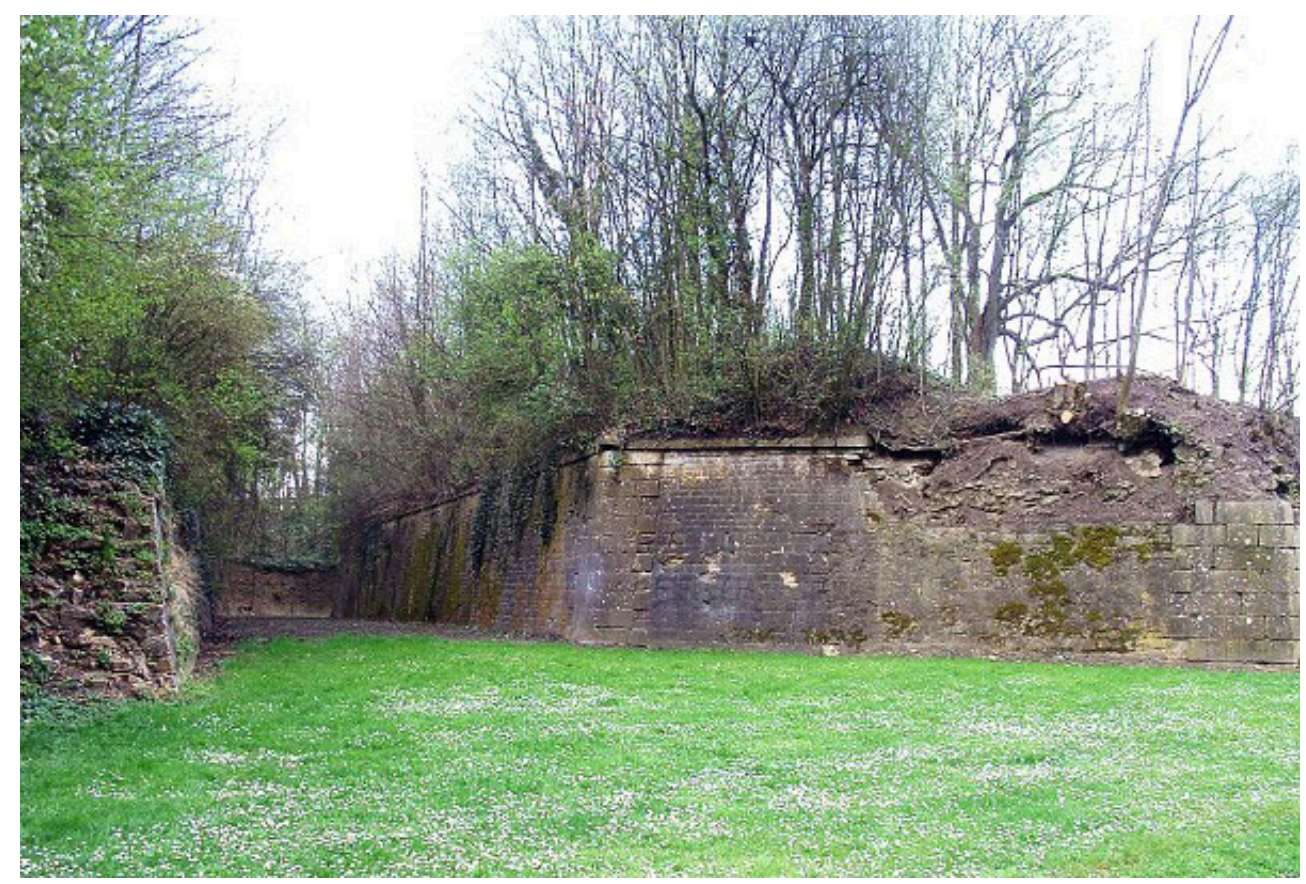

Source : structurae.info

Les exemples les plus pertinents dans la région messine sont ceux de l'ouvrage fortifié du Mont-Saint-Quentin et de la BA 128. Classé en 1994 site Natura 2000, le Mont-Saint- 
Quentin était un lieu de promenade qui abritait deux groupes fortifiés. Une reconversion est envisagée après l'acquisition du lot par la CA2M au titre de l'euro symbolique : les potentialités paysagères $d u$ site permettaient d'en faire un poumon vert associant jardin, écomusée, chemins de randonnée (Cetoba BET Structure, 2005). Mais les travaux de dépollution commencés en 2010 sont interrompus en janvier 2013 pour protéger les chauves-souris et les orchidées identifiées sur le site. Cet arrêt est un coup dur pour la CA2M, qui supporte le projet depuis 10 ans, et notamment pour le maire du Ban-Saint-Martin, Henri Hasser, qui voyait dans la réhabilitation du site un levier de développement puissant pour sa commune.

La BA 128, quant à elle, prend la forme d'un site de 378 hectares situé sur les communes d'Augny et de Marly, près de $7 \%$ de la superficie des deux communes. Sa fermeture entraine le départ de 1250 civils et militaires, soit $8 \%$ de la population. Fermée depuis 2 ans, sa vaste superficie empêche l'émergence d'un projet de reconversion viable au regard de la situation économique et financière des deux communes sur lesquelles elle est implantée (une seule zone d'activité sur la commune d'Augny). Or, sans projet de reconversion, toute cession est impossible. Et trop ancienne pour qu'une remise aux normes soit envisageable (ni éclairage public, ni route, un réseau d'assainissement vétuste), la BA 128 est un no-man's-land dont personne ne veut et dont chacune des deux parties, Ministère de la Défense contre communes, accuse l'autre des surcoûts de chauffage et de gardiennage, en attendant qu'une décision soit prise.

La reconversion des emprises militaires dans l'agglomération messine dépend donc de deux dynamiques : les exigences de l'armée en matière de défense nationale qui définissent l'implantation des emprises militaires sur le territoire métropolitain ; et les relations entre communes de la CA2M qui, lorsqu'elles s'entendent, inscrivent le projet dans les mutations de l'espace urbain. En effet, le devenir du terrain militaire n'est pérenne que si le quartier recommence à vivre avec la ville et respecte les orientations déjà prises par la collectivité locale. Reconvertir une emprise militaire exige donc une réflexion globale d'urbanisme et d'aménagement du territoire.

\section{Les nouveaux usages urbains du patrimoine militaire}

\section{A. La mise en place d'une stratégie immobilière au Ministère de la Défense}

Avec plus de 250000 hectares de terrains, le Ministère de la Défense est le 2e domaine foncier de l'Hexagone. Ses biens constituent un patrimoine hétérogène d'une la valeur de 15 milliards d'euros au 31 décembre 2011 et dont l'entretien coûte près 1 milliard d'euros par an (MRAI, 2013). Jusqu'aux années 1950, la conservation des emprises militaires constituait la ligne directrice de gestion du patrimoine, entrainant un «empilement d'espaces militarisés» sur le territoire français (Mathis, 2011), et l'émergence d'un réseau de villes dans lesquelles les bâtiments militaires occupent des dizaines d'hectares. Dans ce contexte, la restructuration des armées a entrainé le départ de 320000 personnels civils et militaires, rendant désormais inutiles des casernements en centre-ville et des grands équipements (parcs de véhicules et de matériel), soit 6335 hectares d'emprises que les communes doivent réaffecter à d'autres usages ${ }^{4}$. En conséquence, les relations entre l'institution militaire et les décideurs 
urbains se recomposent. La ville devient le lieu d'affrontement entre les logiques des armées, qui s'appliquent à l'échelle nationale, et les logiques des collectivités, qui s'appliquent à l'échelle locale (Dubois-Maury, 1998). La coopération entre les acteurs concernés est donc essentielle pour mener à bien les reconversions et régénérer les tissus urbains.

21 La politique immobilière du Ministère de la Défense répond à la fois aux impératifs de défense nationale et aux exigences de la réforme de la politique immobilière de l'État, selon laquelle l'implantation des administrations ne dépend plus d'un souci de prestige mais d'une préoccupation de fonctionnalité et de rigueur budgétaire. À cet effet, France Domaine est l'unique propriétaire et gestionnaire du patrimoine immobilier de l'État. Ce service créé en 2007 remédie à l'enchevêtrement des compétences entre les autorités chargées des politiques immobilières des différents ministères en devenant l'unique interlocuteur auquel s'adresser en cas de cession d'un bien immobilier de l'État. ${ }^{5}$ La mise en œuvre de cette politique immobilière s'articule autour de l'élaboration d'un Schéma Directeur Interarmées de Base de Défense, établi sur leur périmètre géographique. Il actualise l'évolution de l'ensemble des emprises utilisées par le Ministère de la Défense et s'insère, après avis du préfet de région, dans les PLU. La mise en place d'un tel outil signale une volonté de coordination des échelles : il donne la possibilité aux territoires concernés de planifier des opérations d'aménagement compatibles avec l'évolution du domaine foncier et immobilier du ministère. Seulement, dans le cadre des restructurations de 2008, les mutations foncières sont trop rapides pour les communes puissent être réellement proactives.

\section{B. La cession, préalable administratif nécessaire à la reconversion}

En conduisant la restructuration des armées, le Ministère de la Défense renouvelle sa place dans les territoires. Il développe donc un savoir-faire dans les domaines fonciers et immobiliers et s'ouvre sur des partenaires extérieurs à l'institution. Cette mission est menée conjointement par la Direction de la Mémoire, du Patrimoine et des Archives (DMPA), responsable de l'ensemble de la politique immobilière ; et du Service d'Infrastructure de la Défense (SID), en charge de l'adaptation de l'infrastructure.

La Mission pour la Réalisation des Actifs Immobiliers (MRAI), créée en 1987 et rattachée à la DMPA, est chargée de mettre en oeuvre les compétences techniques et juridiques nécessaires à la cession des emprises militaires. Intervenant dès que l'aliénation d'un bien est prononcé, elle mène l'ensemble des négociations pour conclure un accord avec un acquéreur. Pour ce faire, la MRAI mobilise l'ensemble des autorités civiles et militaires autour d'un comité de pilotage présidé par le préfet, qui sélectionne un cabinet d'ingénierie urbaine en charge de présenter un ou plusieurs scénarios d'aménagement dégageant une potentialité de reconversion du site. Ces projets sont réalisés par des cabinets extérieurs par souci de transparence dans un contexte de polémique sur les potentiels bénéfices que ferait l'armée grâce à la vente de ses terrains (Guelton, 2001).

24 Contrairement à la plupart des transactions immobilières, ce n'est pas le prix du foncier et la localisation qui conditionnent le prix de vente de l'emprise, mais les potentialités de reconversion du site. Sachant qu'aucune emprise militaire ne peut être vendue sans que ces projets aient été proposés auparavant, les biens militaires sont des paquets complexes dont le prix de cession ne reflète pas seulement la valeur vénale en 
fonction de l'évolution des marchés immobilier et foncier (Leroux, 2001). Cela explique la formation de lots dans lesquels l'armée cède à un prix raisonnable des emprises aux potentialités de valorisation très limitées (champs de manoeuvre aux marges de l'agglomération), en les intégrant dans la cession d'emprises à fort potentiel de valeur ajoutée (caserne intégrée à la ville). A Metz, une partie de l'ancienne fortification Cormontaigne, située aux franges est du centre-ville, sur la colline de Bellecroix, est difficile à reconvertir. À ses pieds, le boulevard de Trèves est une friche composée de 4 casernes et traversée par une voie rapide. Ces deux emprises ont été vendues dans le même lot que le centre des essences de Metz-Woippy, l'Arsenal III et le domaine de Mercy. Aujourd'hui, le premier site abrite le dépôt du futur bus interurbain Mettis, le deuxième a une fonction touristique et le troisième accueille le nouveau Centre Hospitalier Régional de Metz-Thionville. Le lot a été vendu à la CA2M pour un total de 2 millions d'euros : le domaine de Bellecroix et le boulevard de Trèves ont été vendus parce qu'ils appartenaient au même lot que le domaine de Mercy, dont la valeur est supérieure.

Une autre solution est la cession à l'euro symbolique, adoptée dans le cadre des mesures de restructurations de 2008 : accordée par décret sur demande expresse des collectivités territoriales, elle leur permet d'acquérir l'emprise sans paiement d'aucune indemnité ou perception de taxes. ${ }^{6}$ Mais la gratuité du bien ne se justifie que dans la mesure où les collectivités réalisent des opérations favorisant la redynamisation économique ou sociale du territoire (DAR, 2010).

Différentes étapes ponctuent les procédures de cession d'une emprise (fig. 2), au cours desquelles interagissent les services militaires chargés des affaires foncières et collectivités locales. Deux types de procédures se sont suivis au cours des 40 dernières années. Le premier type, celui des échanges compensés, domine jusqu'à la fin des années 1970. Il s'agissait d'une relocalisation suburbaine des armées au frais d'une collectivité locale qui, en contrepartie, procédait à la reconversion des espaces libérés (Dubois-Maury, 1998). Cette formule était la plus appréciée dans un contexte de croissance économique et démographique des plus grandes villes de France. À Grenoble, l'urbanisation de l'île Verte dans les années 1960 se fait à mesure que les militaires déménagent à l'extérieur de la ville et cèdent les parcelles dépendantes de la caserne Dode au Conseil Général de l'Isère (Grandvoinnet, 2011). Lyon-Part-Dieu (21ha en 1961), Montpellier-Polygone (11ha en 1972) sont des opérations d'aménagement urbain ayant aussi eu lieu suite à l'échange d'une emprise militaire contre une autre.

Le deuxième type est celui des cessions, utilisé dans les procédures d'aliénation actuelles. Les biens appartenant à l'État relèvent du domaine public et sont inaliénables et imprescriptibles (art L.3111-1 du Code Général de la Propriété des Personnes Publiques in BPOLD, 2012b). Ils doivent donc sortir du domaine public et entrer dans le domaine privé de l'État pour être cédés. La sortie du domaine public se fait par le biais de deux actes administratifs : la désaffectation, qui déclare l'inutilité du bien, et le déclassement, qui le fait passer du domaine public au domaine privé (BPOLD, 2012a). Dès l'aliénation prononcée, l'emprise peut être vendue. Le commandant de la BdD concernée saisit le service France Domaine, seul habilité à signer l'acte de vente. La MRAI avise alors le préfet de département de la décision de céder le bien, procède aux études de reconversion, envoie à France Domaine son évaluation financière, puis mène les négociations (SGA, 2013). 
Figure 3 : L'organisation de la cession d'un terrain militaire

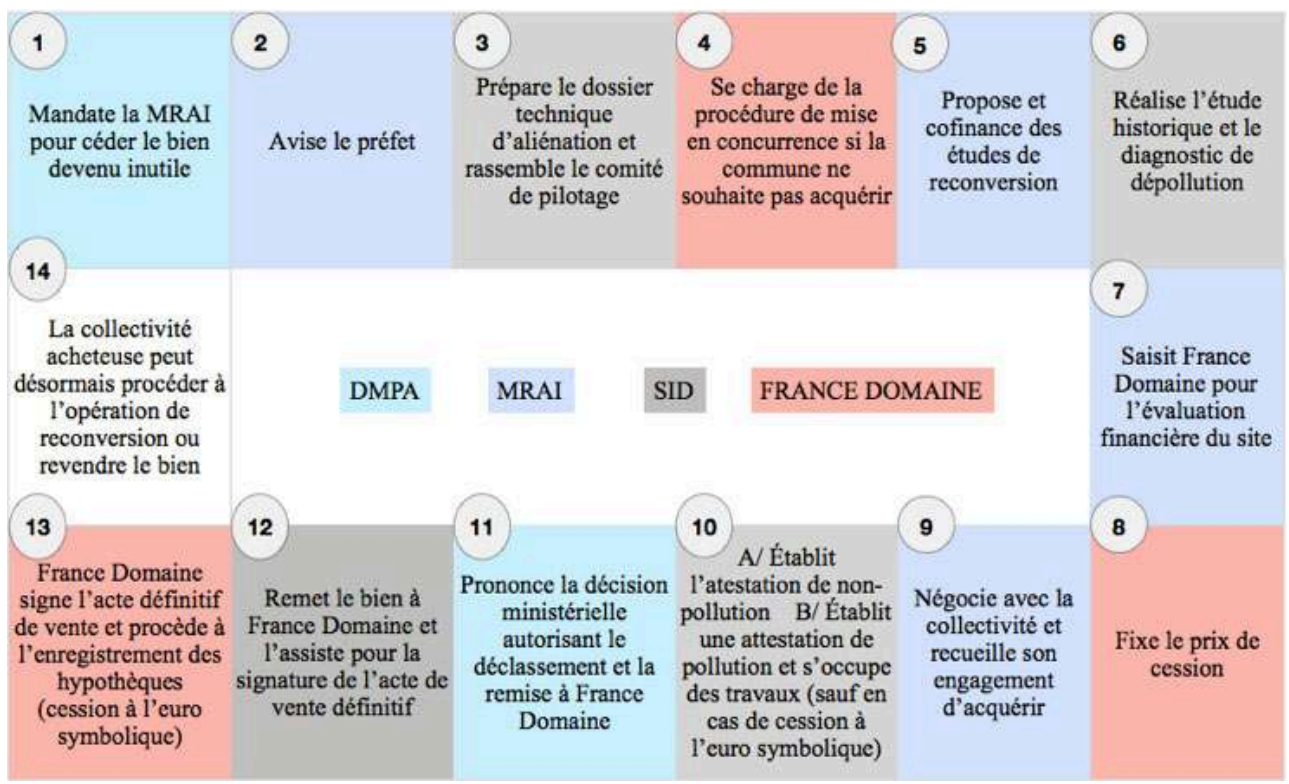

Source : BPOLD, 2012b ; réalisation : Marie-Caroline Gaymard, 2013

\section{Une nouvelle répartition des rôles entre les armées, les collectivités locales et les services de l'État}

Le retrait de l'État impose aux communes de prendre en main l'aménagement de leur territoire. Mais après le départ de l'armée, la demande n'est pas toujours spontanée et immédiate, d'autant que le prix de cession reste le plus souvent élevé. Les repreneurs potentiels cherchent à mener une bonne opération spéculative et rentabiliser leur achat, notamment lorsque l'emprise est située en centre-ville et donne lieu à une revente $d u$ foncier supérieure au prix du marché. C'est pour cette raison que les communes mènent un lobbying auprès de la MRAI et des comités de pilotage, afin de donner priorité aux acquéreurs dont les projets ont une chance de contribuer aux objectifs de développement local : tout projet peu solvable est écarté. À Metz, l'ouverture d'un musée de l'histoire militaire sur l'emprise du boulevard de Trèves a ainsi été mise de côté par rapport à la création de logements de standing. Un autre moyen pour les communes d'orienter le choix des repreneurs est d'user du pouvoir règlementaire de la mairie sur les permis de construire et les contraintes d'occupation des sols (Guelton, 2001).

Mais lorsque la MRAI trouve les propositions inadaptées, la commune est contrainte d'acquérir. Il faut ainsi parfois plus de dix ans pour trouver un projet susceptible de se concrétiser : cet obstacle est le frein principal à la vente de la BA 128, qui stagne depuis déjà 5 ans. Dans le cas d'environ $50 \%$ des sites militaires et $70 \%$ des surfaces libérées, les communes ont assuré le financement de la propriété foncière et de la reconversion (Guelton, 2001). Or, le prix d'acquisition du terrain, parfois très élevé, impose aux communes de trouver un relais financier. Celui-ci est assuré par le biais du Conseil Général ou d'une institution intercommunale, qui va assurer le portage foncier de la reconversion. Dans l'agglomération messine, l'ampleur des terrains à reconvertir rend courant ce recours. Il est assuré par l'Établissement Public Foncier de Lorraine (EPFL), 
créé en 1973 pour alimenter l'offre de terrains destinés à l'implantation d'activités dans toute la région. Sont éligibles les sites militaires dont la dépollution pyrotechnique a été effectuée et dont la cession par le Ministère de la Défense ou l'engagement d'acquérir envers ce dernier a été contractualisé (Soutif et al., 2009). Le recyclage des sites militaires représente ainsi près de $15 \%$ des opérations de reconversion menées par l'EPFL $^{7}$. Les institutions publiques prennent donc en charge des sites que le secteur privé est réticent à investir à cause de coûts de gestion élevés ou de contraintes techniques et administratives dissuasives (temporalité des processus de vente, bâti spécifique).

Les raisons sont aussi politiques : la commune est fortement sollicitée par ses administrés à s'intégrer dans des mutations considérées comme un enjeu municipal (Guelton, 2001). En contrepartie, elle entend jouer un rôle d'arbitrage dans la reconversion des sites, et aussi utiliser les règlements d'urbanisme comme arme défensive face au Ministère de la Défense à cause de "conflits d'aménagement " récurrents (Subra, 2007). En rompant l'isolement physique et conceptuel du site militaire pour le réintégrer dans la vie urbaine, la commune fait valoir sa capacité à intervenir sur le territoire. De son côté, la Communauté d'Agglomération de MetzMétropole se place dans une situation de défense des intérêts locaux et de portage de projet face à l'inertie et à la violence des rapports entre communes et Ministère de la Défense. La BA 128 et le Mont-Saint-Quentin illustrent parfaitement dans quelle mesure «l'aménagement fait conflit» (Subra, 2007). La CA2M réagit à une décision extérieure de l'État, par une série d'actions soit offensive (Mont-Saint Quentin) soit défensive (BA 128). Dans le premier cas, les acteurs territoriaux (collectivités et élus) cherchent à s'approprier l'aménagement du territoire pour y localiser un pôle de loisirs et de tourisme. Dans l'autre cas, la CA2M porte les résistances locales contre le «déménagement du territoire», c'est-à-dire la rationalisation des implantations des armées. La friction continuelle entre les parties prenantes tient à l'évolution des rapports de forces entre les acteurs de l'aménagement du territoire. En effet, les lois de décentralisation des années 1980 ont constitué une rupture. Le rôle de l'État a diminué, marquant le passage d'une situation où il exerçait un contrôle presque absolu sur les politiques d'aménagement à une situation paradoxale : il n'a pas renoncé à son pouvoir sur les décisions stratégiques, comme le montre la restructuration militaire de 2008, mais il ne souhaite pas non plus être un acteur clef du devenir de ces territoires, laissant la place aux collectivités locales. On aboutit de fait à un "partenariat conflictuel» (Subra, 2007). La CA2M coopère d'un côté avec l'État, principal financeur des projets de reconversion, et de l'autre côté elle élabore une vision partagée du territoire avec les 40 communes qui la constituent, pour les mobiliser face aux décisions de l'État.

31 L'ampleur des restructurations de 2008 a de fait conduit à l'État de mettre en place des mesures de soutien financier spécifiques, pour que les communes puissent absorber la libération de dizaines d'emprises militaires sur leur territoire. Par une circulaire du Premier ministre en date du 25 juillet 2008, le gouvernement a ainsi mis en place des dispositifs compensatoires pour contrer les effets négatifs dus aux restructurations (Ginet, 2011). À Metz, ces outils sont essentiels car la restructuration a fait naitre chez les élus un sentiment d'amertume et de délaissement très forts, conduisant à d'âpres négociations avec les représentants du Ministère de la Défense, les uns cherchant à ne 
conserver que le nécessaire pour l'accomplissement des missions, les autres souhaitant relancer un marché immobilier stagnant et conserver des finances correctes.

Les Contrats de Redynamisation des Sites de Défense constituent la mesure la plus lourde. La procédure s'applique pendant trois ans, reconductible une fois pour deux ans. L'enveloppe budgétaire 2009-2015 est de 225 millions d'euros partagés entre une trentaine de sites. Le CRSD de Metz a été signé le 8 juillet 2010 entre le préfet, la région, le département, la CA2M et les maires des communes concernées. Il compense les conséquences de la réforme de la carte militaire, qui s'ajoutent aux fermetures de grands sites industriels fragilisant déjà l'équilibre territorial de la région (DAR, 2010). Selon le Conseil économique et Social de Lorraine, les pertes d'emplois dues aux baisses d'activités des entreprises travaillant en lien avec l'armée représenteraient 1 à 3 emplois par poste supprimé, l'impact démographique étant évalué à une perte de près de 12000 personnes sur l'ensemble de l'agglomération ${ }^{8}$. Pour compenser cela, l'État contribue à hauteur de 32 millions d'euros dans un partenariat à long terme État/ collectivités signataires, et participe d'un effort additionnel de 20 millions d'euros en faveur des grands projets universitaires lorrains. Si les 3 premiers axes du CRSD sont relativement communs (création d'emplois, revitalisation économique, construction d'équipements), le 4e témoigne d'une volonté d'extension urbaine contrôlée par la programmation des reconversions des sites militaires. L'État et les collectivités financent aussi l'offre culturelle dans une logique d'attractivité du territoire : il s'agit alors de réparer le dommage subi en créant les conditions d'un redémarrage d'activités de substitution. Le CRSD à Metz a ainsi permis le financement du Centre PompidouMetz. Son ouverture en mai 2010 a marqué le moment phare d'une grande opération de marketing urbain lancée par l'arrivée à la tête de l'instance municipale de Dominique Gros en 2008. La municipalité dépasse ici les opérations d'aménagement lancées au gré d'opportunités foncières et immobilières décousues, liées au départ des armées, pour s'engager dans un projet de rénovation identitaire qui se fonde sur un renouveau culturel. Cette volonté se traduit par des préoccupations d'esthétique urbaine, avec la mise en place d'un nouveau mobilier urbain confié à un designer réputé (Ruedi Baur) ou le recours à des architectes de renommée internationale dans la réalisation des équipements publics (Ricardo Boffil pour l'Arsenal, Shigeru Ban et Jean de Gastines pour le Centre Pompidou-Metz) ${ }^{9}$. L'opportunité foncière induite par la libération de nombreuses emprises militaires et par leur requalification sont dorénavant saisies pour entrainer la mutation globale d'un quartier et marquer l'avènement d'une identité territoriale originale. Le marketing urbain se fait dans un jeu sur cette ambiance duale, entre atmosphère militaire et esthétique hi-tech (Gravari-Garbas, 1998).

Les Plans Locaux de Redynamisation concernent les sites moins affectés, enregistrant un déficit de 50 à 200 emplois. Cette procédure d'aide déconcentrée mobilise, à l'instar des CRSD, une enveloppe affectée par le préfet de département après concertation entre collectivités locales et autorités militaires. D'autres dispositifs d'accompagnement à l'échelon local sont également mobilisables : les Aides à Finalité Régionale sont destinées à encourager les entreprises à développer des projets de développement. À Metz, sur le plan fiscal, le manque à gagner direct sur les seules taxes d'habitation et la DGF aux communes dépasserait 3,5 millions d'euros par an depuis 2011. Le Fonds pour les Restructurations de la Défense (FReD) et le Fonds National d'Aménagement et de Développement du Territoire complètent l'outil de 
redynamisation du territoire. Depuis 2009, les crédits du FReD ont ainsi subventionné 31 entreprises en Lorraine, pour 3,4 millions d'euros, créant 682 emplois.

Il existe donc une pluralité d'acteurs issus des sphères locales ou nationales, engagés à divers degrés de responsabilité dans les réflexions sur le devenir du site militaire.

À supposer qu'une démarche de cession puis de requalification se mette en place, quatre types de parties prenantes interviennent. Tout d'abord, les acteurs du développement foncier cherchent à optimiser les flux qu'ils contrôlent et mettent de côté la dimension patrimoniale du site qui ne leur profiteront qu'indirectement et à long terme : un des quatre bâtiments du boulevard de Trèves reste à l'état de friche, le temps que le retour sur investissement des travaux précédents soit positif. D'autre part, les acteurs du système de conservation agissent de façon détachée par rapport au niveau local : le Ministère de la Défense a une vision nationale du patrimoine et gère les politiques de manière centralisée. Autres encore sont les acteurs économiques (entreprises, artisans, associations) qui poussent à l'utilisation croissante du patrimoine au profit des usagers extérieurs, dans une logique de développement de l'attractivité de la ville et du territoire. Enfin, les responsables de la planification urbaine se placent dans une position de médiation et définissent, en accord avec les exigences du ministère de la Défense, ce qui est intéressant de préserver, réutiliser ou transmettre.

\section{Le patrimoine militaire sur la scène urbaine}

Après la décision de cession et de libération des emprises, les collectivités publiques doivent assumer toute une série de tâches (mobilisation du foncier, démolitions, mise à niveau des équipements) préalables à l'intervention des acteurs privés, qui agissent alors comme des promoteurs-constructeurs à l'échelle de la parcelle ou de l'îlot urbain. Les mutations à l'intérieur des tissus urbains se font ainsi selon deux modalités : celles qui s'opèrent de manière progressive dans le cadre du réseau viaire et parcellaire déjà existants et celles qui impliquent des changements d'affectation (Novarina et Pucci, 2005).

\section{A. Penser la reconversion à l'échelle d'un bâtiment}

La reconversion des bâtiments militaires a une nature hybride compte tenu de la diversité des emplacements et des aménagements internes (Guelton, 2001). Sous la pression des règlements, des normes ou des usages, la reconversion du site militaire ne se concentre pas seulement sur les bâtiments et le terrain en eux-mêmes mais entraine aussi un effort de revalorisation de tout le quartier. L'action minimale porte sur les réseaux d'eau et d'électricité, qu'il faut raccorder car l'armée dispose des siens propres ; et sur les voiries, qu'il faut relier aux autres quartiers. Les abords du bâtiment bénéficient de programmes de rénovation des façades et du mobilier urbain.

Les opérations de reconversion des bâtiments militaires à Metz relèvent d'un choix entre diverses options sectorielles que sont la consolidation de services publics locaux (Boulevard de Trèves accueillant les services du Conseil Régional de Lorraine, Corps de Garde accueillant l'office de Tourisme), le logement (Caserne Grandmaison) et l'enseignement (Caserne Bridoux, collège Barbot, campus universitaire sur l'île du Saulcy). Les nouveaux usages utilitaires sont ainsi privilégiés quand ils constituent une 
orientation compatible avec le prestige des bâtiments (Godet, 2007 ; Aumaitre, 2007). Dans la même logique, l'Arsenal, ancienne citadelle militaire, a été réhabilité en espace culturel contenant des salles de spectacle, une galerie d'art. Il s'affirme désormais comme un pilier de l'action culturelle et touristique de la CA2M (Fagnoni, 2009).

Photo 2 : L'ancien Corps de Garde sur la place d'Armes, reconverti en Office de Tourisme

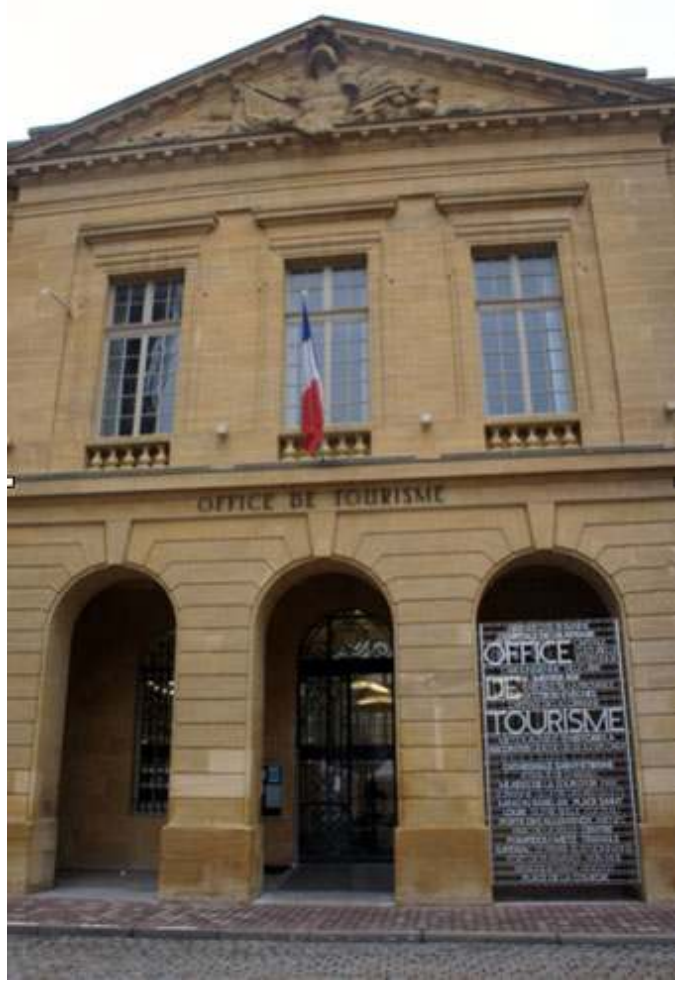

Cliché : Marie-Caroline Gaymard, 2013 
Photo 3 : L'ancien Arsenal, reconverti en salle de concert

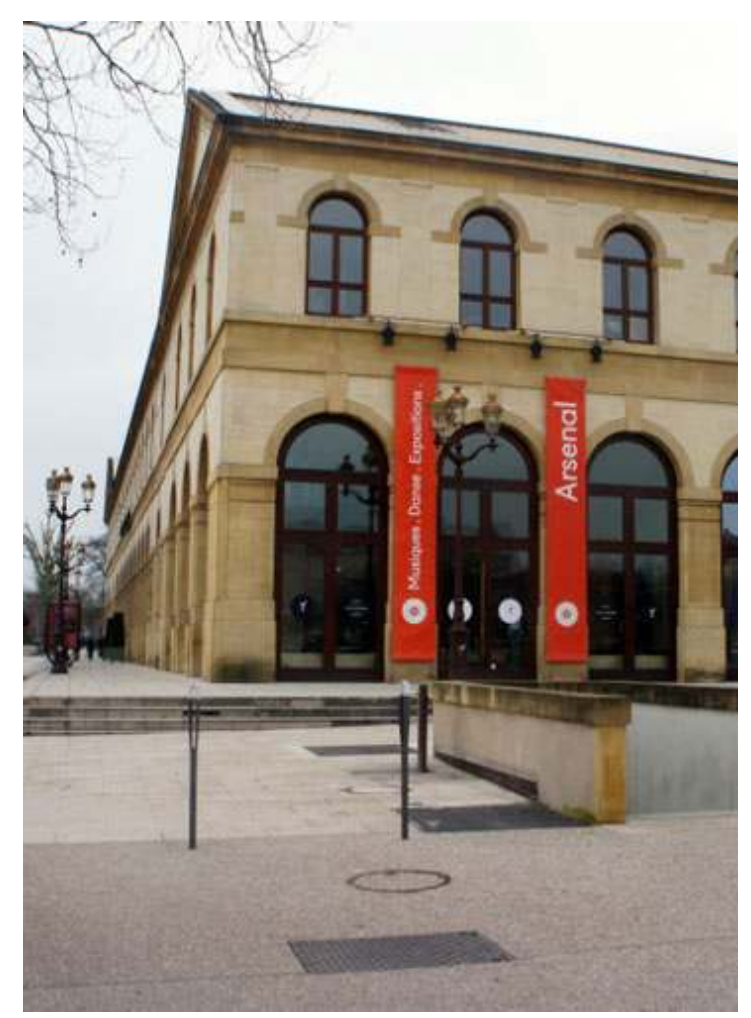

Cliché : Marie-Caroline Gaymard, 2013

\section{B. Penser la reconversion à l'échelle d'un quartier}

La proximité des équipements urbains existants et d'une desserte par les transports en commun sont des atouts qui contribuent à amortir l'investissement nécessaire pour réaliser les dépollutions puis adapter les infrastructures et les réseaux aux nouveaux usages urbains. Ce type d'opération consiste à aménager un quartier sur le site de l'ancienne emprise et développer l'agglomération (Dubois-Maury, 1998), vers le sud et l'est dans le cas de Metz. Certaines opérations ont pour but est renforcer la centralité d'un quartier déjà existant par la création d'équipements collectifs destinés à répondre au développement de nouveaux besoins (caserne Desvallières). D'autres prennent la forme d'un projet urbain de grande ampleur, qui consiste à créer un morceau de ville (cas du boulevard de Trèves).

La reconversion de la caserne Desvallières repose sur un triple enjeu. D'une part, il s'agit de créer de l'habitat en milieu urbain en lien avec les objectifs du Programme Local de l'Habitat et de l'Agenda 21 de la ville et d'éviter la friche urbaine, lourde de conséquences sur l'image du quartier et le prix du foncier environnant. D'autre part, il faut reconnecter le quartier de Devant-lès-Ponts au centre-ville en créant un nouvel accès à l'îlot urbain du côté ouest et en élargissant la voirie du côté est, afin d'optimiser la proximité des axes de communication majeurs comme l'A31 et l'A4. Enfin, il est question de préserver une partie du patrimoine architectural de la caserne, élément majeur de l'histoire du quartier auxquels les Messins tiennent particulièrement. Alors que la démolition de l'ensemble du quartier était originellement prévue, le jeu de la démocratie participative a permis aux habitants de faire valoir leur lien sentimental 
avec ces bâtisses militaires de la fin du XIXe siècle. Le nouveau projet intègre désormais la conservation de toute la partie sud du quartier.

41 Concernant le boulevard de Trèves, ensemble de cinq bâtiments en pierre de Jaumont étalés sur une longueur de $400 \mathrm{~m}$ et encadrant une voie rapide très fréquentée, il s'agit de mettre fin à la rupture géographique du quartier avec le centre-ville (Cadoux, 2007). La Seille est une première rupture physique, doublée par la voie ferrée et complétée par la voie rapide, tandis que les HLM construits sur les hauteurs du fort de Bellecroix, en retrait des fortifications, forment une rupture sociale et identitaire par rapport à un centre-ville plus bourgeois (la partie sud et l'avenue Foch) et plus ancien. Requalifier la caserne vise à développer l'agglomération vers l'est. C'est ainsi qu'on trouve aujourd'hui sur le site des commerces qui couvrent les secteurs de l'alimentation (marché bio), de la restauration (McDonald's), de l'équipement de la maison et des loisirs ; mais aussi un plateau médical, des bureaux et des logements de standing dont le prix élevé par rapport aux habitations environnantes s'explique par la proximité du centre-ville, la présence d'une voie rapide et la valeur historique du bâtiment. L'ensemble cherche à répondre au concept urbain de «nouveau quartier» (Bourdin, 2003) : il est facile d'accès, facile d'usage et sait se mettre en scène. Il ne se définit pas par une «identité» issue de sa localisation dans l'ordre spatial plus large de la ville (comme le quartier de Plantières-Queuleu au sud pour la caserne Grandmaison) ; mais par un "paysage urbain». Ce dernier résulte de l'immense effort développé par les urbanistes de la ville pour créer une ambiance sur le site reconverti (espaces publics ouverts, gamme de mobilier urbain moderne...).

Photo 4 : Un des bâtiments de la caserne du boulevard de Trèves, avant reconversion en 2007 (en haut) et après reconversion en 2013 (en bas)

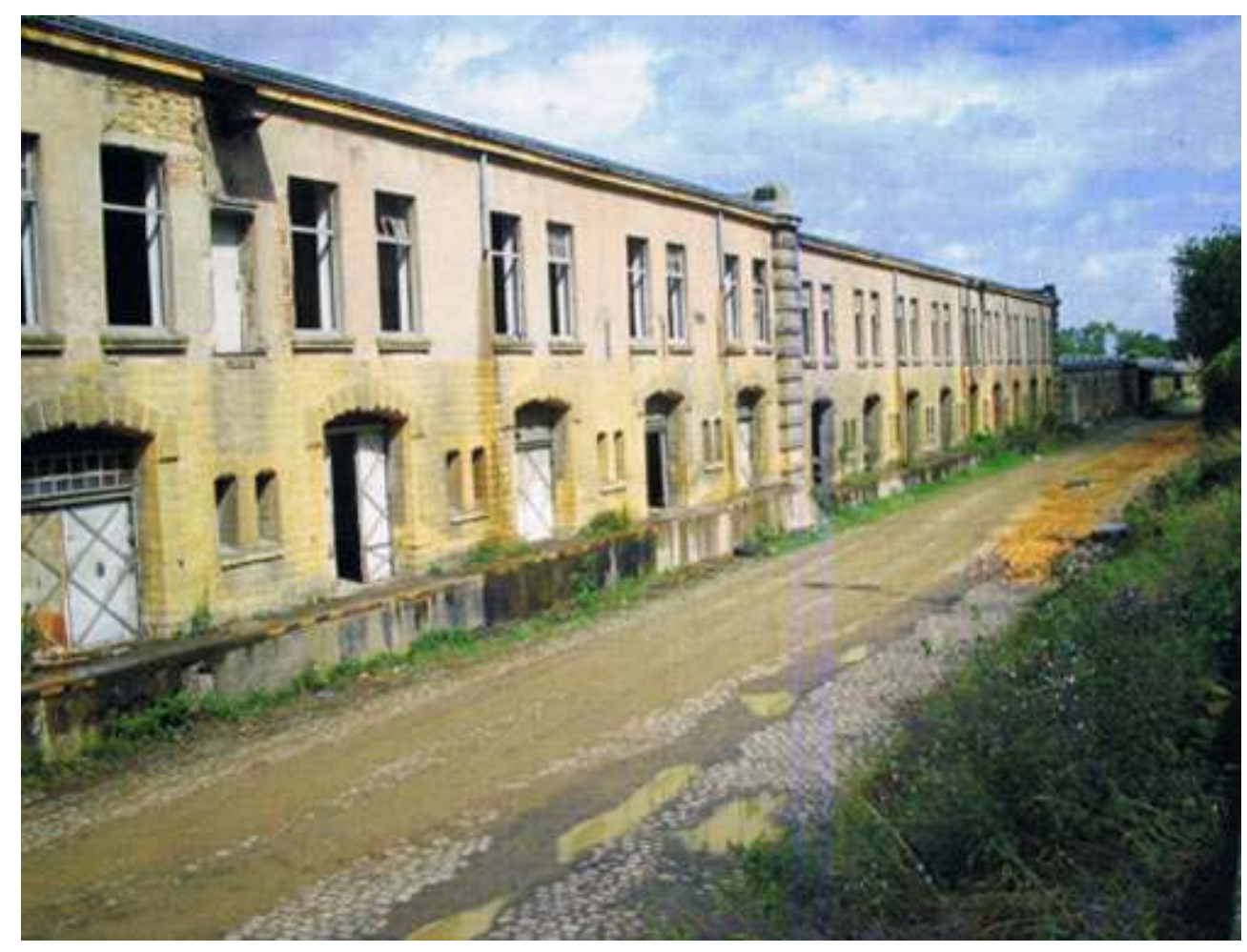




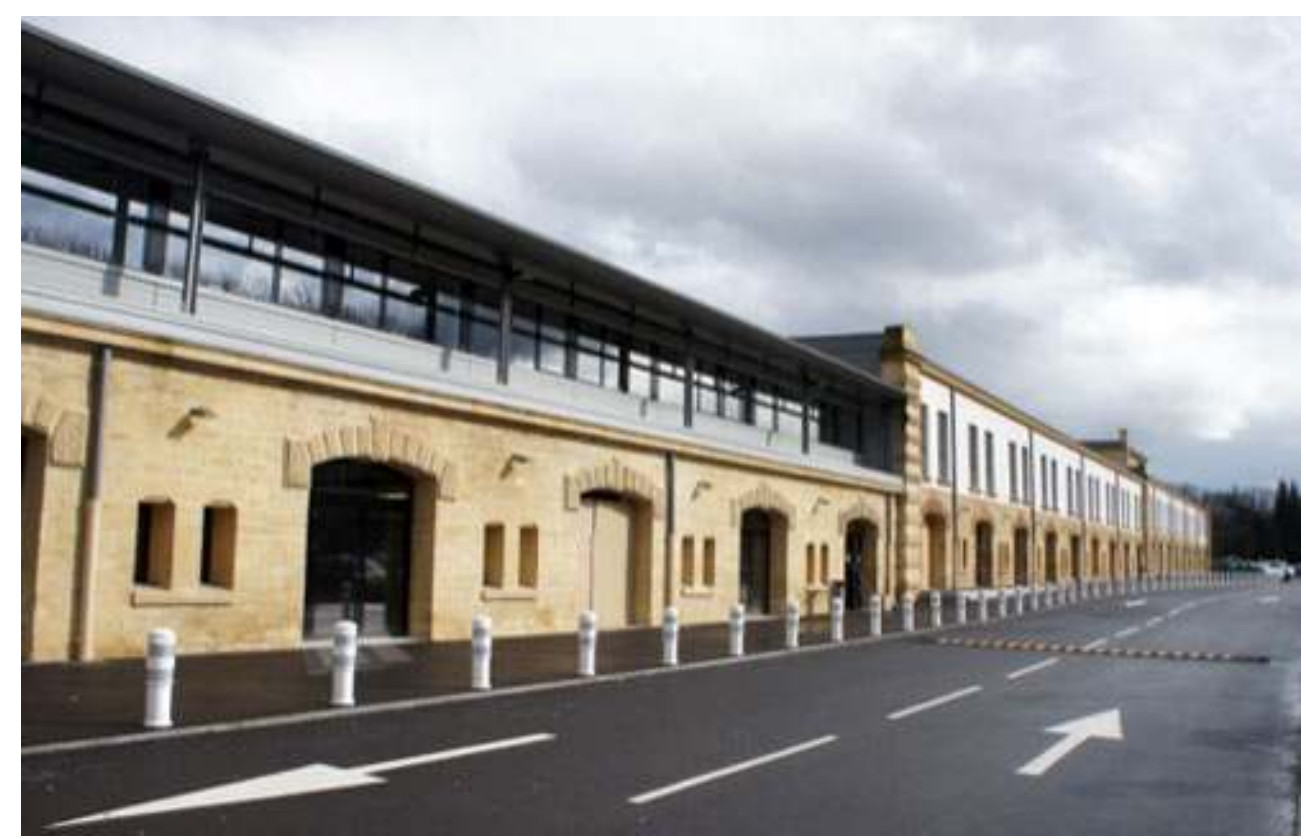

Clichés : P. Rausch, 2007 (en bas), Marie-Caroline Gaymard, 2013 (en haut)

Les impacts morphologiques et fonctionnels des réaffectations des sites militaires varient donc considérablement d'une opération complexe équivalente au réaménagement d'un quartier à une opération ponctuelle de reconversion d'un bâtiment, comme le montre la typologie indicative des reconversions ci-dessous. Les effets, en termes de régénération de la base économique, ne peuvent encore être mesurés de par le caractère récent des opérations. Ces effets dépendent aussi de la capacité des villes à intégrer la reconversion dans un programme de développement plus général comme le fait Metz avec la métropolisation annoncée de l'agglomération messinne et du Sillon Lorrain. Aussi, alors qu'elle abandonne bon nombre de sites qu'elle occupait au centre des villes, l'institution militaire est en passe de renouer avec une certaine visibilité urbaine. 
Tableau 1 : Typologie indicative des opérations de reconversion menées en France au cours des 40 dernières années

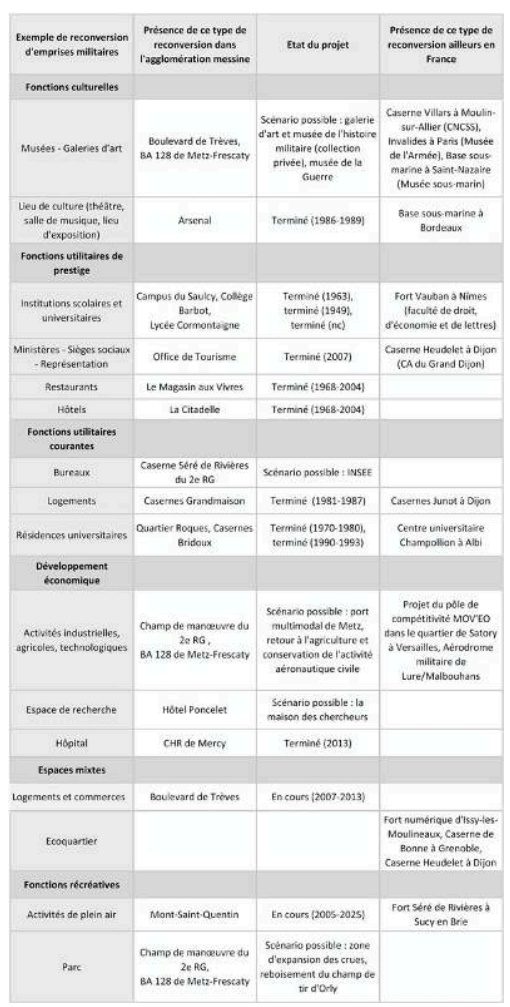

Conception-réalisation : Marie-Caroline Gaymard, 2015

\section{Conclusion}

Par-delà les procédures administratives qui mettent en présence les services du Ministère de la Défense et les collectivités territoriales, une constatation s'impose sur l'ampleur des mutations d'usage et de régime qui affectent le foncier urbain de l'agglomération messine, suite au désengagement de l'appareil militaire depuis les restructurations de défense de 2008 et la mise en place de la RGPP. Une libération foncière aussi vaste conduit à un rejet des sites de la part de la municipalité qui, loin de les considérer comme des atouts, est contrainte d'intégrer les bâtiments à sa politique de développement urbain par peur de la friche. Le sentiment global des autorités publiques, notamment la CA2M, est celui d'avoir la main forcée par le Ministère de la Défense, qu'elles considèrent comme un organe se pensant autosuffisant par rapport à la sphère civile et suivant sa propre logique d'aménagement du territoire.

Le fait militaire est ancré dans l'espace urbain messin et structure une part notable du vécu des citadins. Au moment où l'armée se retire, toucher à la présence militaire c'est toucher à la ville, ce qui peut justifier la vigueur et l'amertume de certaines réactions. Sans utilité pour l'armée, la valeur économique du patrimoine militaire change, son usage se transforme. Mais les investisseurs privés se mobilisent peu et les collectivités locales se trouvent en charge de l'acquisition des terrains libérés par l'institution militaire, devant supporter la plus grande partie des coûts de reconversion. La cohésion des acteurs ne parvient pas à uniformiser les multiples situations d'emprises qui sont menacées de destruction ou d'effacement dans la modernisation du centre-ville, quand 
elles ne représentent pas des centaines d'hectares de jachère en rase campagne dont personne ne sait que faire. Cette absence de considération se retrouve dans la patrimonialisation des bâtiments militaires qui ne s'imposait pas comme une nécessité puisque l'institution militaire était solidement implantée sur le territoire. Le caractère militaire des bâtiments était perçu comme quelque chose d'encombrant, participant d'une image de ville de garnison guère attractive. L'annonce des mesures de restructurations a fonctionné comme un coup de fouet pour la collectivité locale qui a soudainement pris conscience de la valeur du bâti militaire et de la possibilité de l'intégrer aux politiques culturelles et touristiques en cours d'élaboration sur le territoire. Elle est désormais une facette du passé de Metz au même titre que la ville romaine et la république messine. La vague massive de cessions foncières et immobilières a ainsi partiellement remis en cause la place de la métropolisation dans les projets de développement du territoire, avant que la CA2M ne décide de faire de la reconversion des emprises militaires un exemple symptomatique du dynamisme messin et de la capacité de la ville à se renouveler.

Le problème majeur est finalement la divergence entre les logiques des armées et celles des collectivités territoriales, qui fait de la reconversion des emprises militaires une situation de "géopolitique locale » (Subra, 2007). D’un côté, les armées ne conservent que le nécessaire à l'accomplissement de leurs missions, quitte à entrer en conflit avec une commune pour qui la libération des emprises impliquerait une baisse subite du prix du foncier et une opération d'acquisition/d'aménagement très coûteuse. De l'autre côté, les logiques des collectivités territoriales s'inscrivent dans une double préoccupation. Il s'agit en premier lieu de devenir le maitre d'ouvrage d'opérations complexes sur des terrains dont la localisation urbaine est cruciale. En parallèle, elles souhaitent acquérir ces espaces sans obérer durablement les finances locales. Ce travail n'a pas pour rôle de trancher la polémique entre un État qui n'a pas le droit de brader son patrimoine et un Ministère de la Défense qui s'enrichit. La contrainte budgétaire est souvent modulée, le Ministère de la Défense consentant à des abattements à partir de considérations d'intérêts général au bénéfice de la municipalité pour lui permettre de mobiliser les fonds.

Notre étude s'appuie sur la seule agglomération de Metz, mais elle fait ressortir certaines modalités invariantes des politiques de reconversion, et ce dans un contexte économique et social mouvant suivant les villes et les régions concernées par les restructurations militaires. Les reconversions menées sur l'agglomération messine se retrouvent aisément sur d'autres territoires français ou étrangers, mais la particularité de Metz est de toutes les concentrer. À terme, il conviendrait d'étudier les coûts et les conséquences de ces éléments. L'analyse bénéficie de six années de recul par rapport aux évènements de 2008, pendant lesquelles des stratégies ont pu être définies et mises à l'épreuve (renouvellement de mandats politiques locaux, parution d'un nouveau Livre Blanc en 2013, achèvement du calendrier des premières mesures compensatoires).

La dernière vague de réformes, annoncée le 15 octobre 2014 par le ministre de la Défense, Jean-Yves Le Drian, n'amène comme nouveauté pour l'agglomération messine que la seule arrivée de «médiateurs mobilité» dans le cadre du Plan d'Accompagnement des Restructurations. Mais elle montrera peut-être l'exemplarité de Metz et l'avantage qu'il y a à tirer parti de son retour d'expérience en matière de reconversion des emprises militaires. 


\section{BIBLIOGRAPHY}

Aumaitre M., 2007, Nos casernes disparues, Ed. Alan Sutton, «100 dates pour l'histoire», 128 p.

Bonnet J., 1994, Les grandes métropoles mondiales, Paris; Nathan-Université, 192 p.

BPOLD, 2012a, La politique immobilière du Ministère de la Défense, Paris, Ministère de la Défense.

BPOLD, 2012b, Guide des procédures domaniales, Paris, Ministère de la Défense.

Bour R., 2007, Histoire de Metz, Nouvelle Edition, Metz, Editions Serpenoise, 270 p.

Bourdin A., 2003, «Urbanisme et quartier», Terrain, $n^{\circ} 41,13$ p.

Cadoux J.-C., 2007, Boulevard de Trèves, Metz, Manulor \& SAS Trèves Investissement, [études].

Cetoba BET Structure, 2005, Mise en sécurité des sites et ouvrages militaires du Mont-Saint-Quentin (57), Metz, CA2M \& EPF Lorraine, $70 \mathrm{p}$.

Clémenceau C., Férré-Lemaire I., «Adieu l'armée. Le patrimoine de l'armée à Metz» in Urbanisme n²92, 1997, p. 14

DAR, 2010, Contrat de Redynamisation de Site de Défense de Metz et son agglomération, Metz, Ministère de la Défense, $76 \mathrm{p}$.

Dubois-Maury J., 1998, «Impacts urbains des restructurations de l'appareil militaire en France », Annales de Géographie, vol. 107, n 599, p. 88-97.

Fagnoni E., 2009, «Les nouveaux territoires urbains de la culture : Metz et le Centre Pompidou», in Politiques culturelles et enjeux urbains, séminaire du 06/01/2009, ENS Ulm, Paris, 11 p.

Gallas S., Huber S. \& Steinmetz F., 2009, L'impact de la carte militaire sur l'urbanisme à Metz, Metz, Université Paul Verlaine, $21 \mathrm{p}$.

Ginet P., 2011, «Les restructurations militaires en Lorraine : enjeux d'aménagement à différentes échelles», Revue Géographique de l'Est, vol. 51, n²1-2, 16 p.

Godet O., 2007, Patrimoine reconverti, du militaire au civil, Paris, Nouvelles éditions Scala, 267 p.

Grandvoinnet P., 2011, «Les emprises militaires dans l'urbanisme grenoblois du XXe siècle : des opportunités foncières au patrimoine paysager ", In Situ. Revue des patrimoines, n 16, 29 p.

Guelton S., 1998, «Dix années de vente des terrains militaires», Études foncières n 79, p. 19-20.

Guelton S., 2001, «La reconversion des emprises publiques : quelles opportunités pour le développement urbain; le cas des emprises du ministère de la Défense», in FREVille (2001) Renouveler la ville : les enjeux de la régénération urbaine, Actes du séminaire des 17-18 mars 2000, FREVille publications, $\mathrm{p}$ 141-150.

Guelton S., 2001, «Les communes face à la reconversion des terrains militaires», Etudes Foncières $\mathrm{n}^{\circ} 93, \mathrm{p} 22-24$.

Gravari-Barbas M., 1998, « Belle, propre, festive et sécurisante : l'esthétique de la ville touristique », Norois, vol. 178, n 1, p. 175 - 193.

INSEE, 2011, « Diagnostic 2010 de la Moselle : dynamiser la croissance démographique en confortant l'attractivité économique ", Économie Lorraine, n²47-248, 24 p.

Leroux P., 2003, Présence et représentation de l'institution militaire dans la ville, Paris, Centre d'Etudes en Sciences Sociales de la Défense, 270 p. 
Marquis C. et Demange M., 2010, «L'impact des évènementiels touristiques en Lorraine : une évaluation par la fréquentation quotidienne», Économie Lorraine, n²11, INSEE, 6 p.

Mathis D., 2011, «'Quand l'armée s'en va'.Approche géohistorique des restructurations des espaces militarisés : l'exemple du sud mosellan», Revue Géographique de l'Est, vol. 51, n¹-2, 15 p.

Merchet J.-D., 2005, Les transformations de l'armée française, Hérodote, 2005/1, nº116, p. 63-81.

MRAI,2013, Rapport d'activité 2012, Paris, Ministère de la Défense, [document word].

Novarina G. et Pucci P., 2005, «Renouvellement en France/Requalification en Italie : formes récentes du partenariat public/privé ",. Les annales de la recherche urbaine, vol. Renouvellements urbains, $n^{\circ}$ 97, p. $83-91$.

Pommier C., 2010, « Patrimoines militaires en milieux urbains: réussites et combats à mener », Art et société, Brest, Presses universitaires de Rennes, 9 p.

$\mathrm{SGA}, 14 / 02 / 2013$, Instruction $n^{\circ} 00302$ relative à la politique immobilière du ministère de la défense, Paris, Ministère de la Défense, 38 p.

Soutif V., Courtin J.-C., Stenger R. \& Henneton I., 2009, Reconversion du patrimoine militaire désaffecté en Lorraine : mise en oeuvre du programme Régional d'Accompagnement des Restructurations de Défense, EPF Lorraine, $36 \mathrm{p}$.

Subra P., 2007, Géopolitique de l'aménagement du territoire, A. Colin, coll. Perspectives Géopolitiques, Paris, $327 \mathrm{p}$.

Truelle C., 1986, Metz, deux mille ans d'architecture militaire, Metz : Ed. Serpenoise, 173 p.

\section{Sites internet consultés :}

Site de l'Établissement Public Foncier de Lorraine, http://www.epfl.fr, 18 septembre 2014.

Site de la ville de Metz, http://www.mairie-metz.fr, 18 septembre 2014.

Site du Service public de la Diffusion du Droit, http://www.legifrance.gouv.fr, 10 juin 2012.

Site du Sénat, http://www.senat.fr , 4 mai 2013.

Site du Ministère de la Défense , http://www.defense.gouv.fr, 4 mai 2013.

Site de la Direction de l'Information légale et administrative, http://www.vie-publique.fr, 4 mai 2013.

Site de la Délégation Aux Restructurations de Défense, http:// www.restructurations.defense.gouv.fr, 4 mai 2013.

\section{NOTES}

1. C. Clémenceau, I. Férré-Lemaire, «Adieu l'armée. Le patrimoine de l'armée àMetz»in Urbanisme n²92, 1997, p. 14

2. site internet de la Direction de l'Information Légale et Administrative

3. Site du Ministère de la Défense

4. Site du Sénat

5. Site du Service public de la Diffusion du Droit

6. Site de la Délégation Aux Restructurations de Défense

7. site internet de l'EPFL

8. site internet de la DAR 


\section{ABSTRACTS}

This research paper deals with the new urban uses of former military lands in Metz, a garrison town heavily affected by the restructuring in the defense sector begun in 2008. Beyond the loss of an important activity, the release of these boundaries is a major asset. Indeed, the opening and conversion of several acres in the city center is an opportunity that can overcome a deficiency habitat or install new equipment. Economic and political stakeholders coordinate among themselves in order to look for a new assignment to military sites no more needed by the army, which is an integral part of the metropolitan reviving momentum and renewed identity the city is experiencing for several years.

L'objet de cet article est de s'interroger sur les nouveaux usages urbains des anciennes emprises militaires à Metz, dans une ville de garnison fortement touchée par les restructurations de défense lancées en 2008. Au-delà de la perte que constitue le départ d'une importante activité, la libération de ces emprises constitue un atout. Le désenclavement et la reconversion d'un terrain de plusieurs hectares en centre-ville est une opportunité qui permet de pallier une carence en matière d'habitat ou d'installer de nouveaux équipements. En accord avec les acteurs économiques et politiques, la recherche d'une nouvelle affectation aux sites militaires devenus inutiles à l'armée participe de l'élan métropolitain et du renouvellement identitaire que connait la ville depuis plusieurs années.

Das Ziel dieses Artikels besteht darin, sich über die neuen Stadtgebräuche der ehemaligen militärischen städtischen Flächen zu fragen. Sie befinden sich in Metz, eine Garnisonsstadt, der in 2008 im einem militärischen Verteidigung- und Umstrukturierungsplan geworfen würde. Jenseits des Verlustes, den die Abfahrt einer wirtschaftlichen Tätigkeit bildet, ist der Verkauf dieser Militärgelände wertvoll. Die Umstellung eines Ortes, das mehrere Hektare in der Stadtmitte beläuft, ist eine Zweckmäßigkeit, die erlaubt, ein Programm für den Bau fester Wohnungen durchzuführen oder neuen Verkehrstrukturen zu errichten. Wirtschaftliche und politische Schauspielern arbeiten zusammen, um ein neuer Verwendungszweck zu finden, in die militärischen städtischen Flächen, die der Armee nutzlos geworden sind. Diese Forschung ist ein wichtiger Bestandteil der hauptstädtischen Entwicklung und der identitätsstiftenden Erneuerung der Stadt, die die Stadt seit mehreren Jahren erlebt.

\section{INDEX}

Schlüsselwörter: Metropole, Metz, militärischer Umstrukturierungsplan, militärisches Erbe, Rehabilitationmaßnahmen, Stadt- und Raumplannung., städtische Umstellung

Mots-clés: aménagement du territoire, métropole, Metz, patrimoine militaire, reconversion, réhabilitation, restructuration de défense

Keywords: adaptative reuse, defence reform and restructuring, metropole, Metz, military heritage, rehabilitation, spatial planning 


\section{AUTHOR}

\section{MARIE-CAROLINE GAYMARD}

Sciences Po Paris, Master d'Affaires Urbaines, «Stratégies territoriales et urbaines» -

gaymard.mariecaroline@gmail.com 